\title{
Estado nutrimental en el extracto celular de pecíolo y hojas de genotipos de jamaica
}

\section{Nutrimental status in the cellular extract of petiole and leaves of genotypes of roselle}

Emilia F. Flores-Bernal ${ }^{1}$ (D)

Manuel Sandoval-Villa2 (D)

María Guzmán-Martínez $z^{3}$

Mariana Espinosa-Rodríguez 1 (D)

Mirna Vázquez-Villamar 1 (D)

Juan E. Sabino-López ${ }^{1 *}$ (1D)

${ }^{1}$ Facultad de Ciencias Agropecuarias y Ambientales, Universidad Autónoma de Guerrero, Periférico poniente S/N Col. Villa de Guadalupe, CP. 40040. Iguala de la independencia, Guerrero, México.

${ }^{2}$ Postgrado en Edafología, Colegio de Postgraduados, Campus Motecillo, Km. 36.5 Carretera México-Texcoco, CP. 56230. Montecillo, estado de México, México.

${ }^{3}$ Facultad de Matemáticas, Universidad Autónoma de Guerrero, Av. Lázaro Cárdenas s/n, CP. 39087. Chilpancingo, Guerrero, México.

*Autor de correspondencia: juanelias_sab@hotmail.com

\section{Artículo científico}

Recibido: 28 de febrero 2021

Aceptado: 07 de octubre 2021

Como citar: Flores-Bernal EF Sandoval-Villa M, Guzmán-Martínez M, Espinosa-Rodríguez M, VázquezVillamar M, Sabino-López JE (2021) Estado nutrimental en el extracto celular de pecíolo y hojas de genotipos de jamaica. Ecosistemas y Recursos Agropecuarios Núm. Esp. II: e2928. DOI: 10.19136/era.a8nll.2928

RESUMEN. Existen varias técnicas de diagnóstico nutrimental en los cultivos, para corregir la fertilización. Pero, al igual que en otros cultivos, en el de jamaica (Hibiscus sabdariffa L.) no se revisa el estado nutrimental. El objetivo de este trabajo fue estudiar el estado nutrimental de ocho genotipos de jamaica en etapa de floración, mediante el análisis en el extracto celular de pecíolo (ECP), lecturas SPAD y tejido vegetal en laboratorio. Se recolectaron y sembraron ocho genotipos de jamaica en bolsas de polietileno de $12 \mathrm{~L}$ llenadas con una mezcla de tezontle y tierra de monte (luvisol) $(1: 1 \mathrm{v} / \mathrm{v})$ en un diseño completamente al azar con cinco repeticiones. Las plantas crecieron en un invernadero cubierto con plástico blanco de $800 \mu \mathrm{m}$ con $70 \%$ de trasmisión de luz. Las plantas se fertilizaron con $44 \mathrm{~N}-30 \mathrm{P}-20 \mathrm{~K} \mathrm{~kg} \mathrm{ha}^{-1}$ y se regaron con agua corriente. Se determinaron las lecturas SPAD en hojas recientemente maduras y en el ECP se determinó la concentración de $\mathrm{NO}_{3^{-}}, \mathrm{K}^{+}, \mathrm{Ca}^{2+}$ y Na${ }^{+}$. Las hojas se procesaron para análisis químico de nitrógeno total, fósforo, potasio, calcio, magnesio, hierro, cobre, zinc, manganeso y boro. Se tuvieron diferencias significativas en los iones para ECP, lecturas SPAD y concentración nutrimental en hojas de los genotipos estudiados. La concentración de nutrimentos de los iones específicos en ECP, lecturas SPAD y en tejido vegetal pueden ser usados como valores de referencia para comparar con estudios posteriores en este cultivo.

Palabras clave: Diagnóstico nutrimental, fertilización, ionómetros, lecturas SPAD, jamaica.

ABSTRACT. There are several nutritional diagnosis techniques in the corps, to correct fertilization. But, as in other crops, in roselle (Hibiscus sabdariffa L.) the nutritional status is not checked. The aim of this work was to study the nutritional status of eight roselle genotypes in flowering stage, by analyzing the petiole cell extract (ECP), SPAD readings and plant tissue concentrations in the laboratory. Eight roselle genotypes were collected and sown in $12 \mathrm{~L}$ polyethylene bags filled with a mixture of tezontle and bush soil (luvisol) $(1: 1 \mathrm{v} / \mathrm{v})$ in a completely randomized design with five replications. The plants were grown in a greenhouse covered with white $800 \mu \mathrm{m}$ plastic with $70 \%$ light transmission. The plants were fertilized with $44 \mathrm{~N}-30 \mathrm{P}-20 \mathrm{~K} \mathrm{~kg} \mathrm{ha}^{-1}$ and watered with running water. The SPAD readings were determined in recently mature leaves and $\mathrm{NO}_{3^{-}}, \mathrm{K}^{+}, \mathrm{Ca}^{2+}$ and $\mathrm{Na}^{+}$were determined in the petiole cell extract with ionometers. The leaves were processed for chemical analysis of total nitrogen, phosphorus, potassium, calcium, magnesium, iron, copper, zinc, manganese and boron. There were significant differences in the ions for ECP, SPAD readings and nutritional concentration in leaves of the genotypes studied. The nutrient concentration of the specific ions in the petiole cell extract, SPAD readings and in plant tissue can be used as reference values to compare with subsequent studies on this crop.

Key words: Nutritional diagnosis, fertilization, ionometers, SPAD readings, roselle. 


\section{INTRODUCCIÓN}

La expresión del potencial de rendimiento de los cultivos depende de varios factores, los que están determinados por su constitución genética, los externos como el clima, características del suelo, condiciones nutrimentales, técnica de producción y factores biológicos (Castro-Brindis et al. 2000). Uno de los factores que limitan el rendimiento de los cultivos son las condiciones nutrimentales, las cuales pueden ser controladas (Sánchez-García et al. 2016). Además, es común la presencia de variaciones en la concentración de los nutrientes de los cultivos, según la especie, variedad, edad del tejido, posición de la hoja, clima, época del año, momento de muestreo y presencia de residuos, que complican el establecimiento de dosis de fertilización adecuadas (Pino et al. 2012).

Por lo anterior, es necesario el conocimiento del estado nutrimental de las plantas (Ribeiro et al. 2015), mediante el estudio de la absorción de nutrimentos, para establecer las bases de la fertilización de los cultivos y ajustar al ciclo productivo y optimizar la fertilización, al mismo tiempo que se evita el deterioro de los suelos y se disminuye el impacto de la fertilización en el ambiente (Noh-Medina et al. 2010). Dicho control de las condiciones nutrimentales en las que se desarrollan los cultivos es posible mediante el diagnóstico nutrimental (Castro-Brindis et al. 2000). Para diagnosticar y verificar que el estado nutrimental de un cultivo sea el ideal en cada etapa de su ciclo de desarrollo, se cuenta con herramientas analíticas químicas que sirven para medir las concentraciones nutrimentales en los tejidos vegetales y en el suelo, para tomar decisiones correctivas en su momento (Etchevers 1999, Sánchez-García et al. 2016).

Una manera tradicional de evaluar el estado nutrimental de las plantas es mediante el análisis químico, en el que se evalúa la composición mineral de todos los órganos de la planta, proporcionando información sobre la demanda total de nutrimentos que toma del suelo, lo que permite conocer las características de su condición nutricional y de su potencial productivo (Noh-Medina et al. 2010). Otro método para evaluar el estado nutrimental de las plantas es el análisis de hojas u órganos de referencia, en donde el órgano usado con mayor frecuencia es la hoja, el cual requiere definiciones precisas en cuanto a la edad, orientación, altura, posición, cultivar y en algunos casos la hora del día en que se hace el muestreo (Etchevers 1999). Pero el principal inconveniente del diagnóstico nutrimental en tejido foliar en laboratorio es que implica todo un proceso de preparación y análisis de la muestra, por lo que requiere de mayor tiempo para la obtención de los resultados; además, los datos obtenidos pueden enmascarar algún problema nutrimental, debido a que puede medirse la cantidad acumulada de este nutrimento y no la cantidad presente en los sistemas de conducción (Castro-Brindis et al. 2000).

Recientemente, la extracción y cuantificación de nutrimentos con medidores portátiles se ha convertido en una herramienta rápida y eficaz, que además tiene menor costo para determinar el estado nutrimental de un cultivo, aumentando la posibilidad de su uso como criterio de evaluación de algunos nutrimentos en las plantas (Ribeiro et al. 2015, ÁvilaJuárez y Rodríguez-Ruíz, 2020). Ante ello, el análisis de extracto de pecíolo (ECP), es una técnica complementaria del análisis químico de tejido vegetal, el cual refleja el nivel de suministro de nutrimentos en las plantas, herramienta que se ha usado para conocer el estado nutrimental y riesgos de salinidad en cultivos intensivos establecidos tanto con fertirriego, en suelo o en sustrato (Cadahía 2008). El análisis de ECP es una técnica de diagnóstico nutrimental de los cultivos en campo, que sirve para establecer índices de referencia nutrimental de los mismos, con los que se pueden establecer ajustes oportunos en el manejo de la nutrición (Gómez et al. 2017). Aunque una desventaja es la dificultad que tiene para interpretar los resultados, en particular, es difícil determinar si un nutriente está presente en exceso o es deficiente y, por lo tanto, afecta el rendimiento (ÁvilaJuárez y Rodríguez-Ruíz 2020). Actualmente, las mediciones de iones en el ECP, es ampliamente utilizada en sistemas intensivos de producción, debido a que presenta buena correlación con el rendimiento, en comparación con los diferentes métodos de diagnóstico de suelo y tejido en laboratorio (Llanderal et 
al. 2020). Dicha técnica podría ser una alternativa práctica para revisar el estado nutrimental de cultivos como la jamaica, situación que puede determinar el rendimiento del mismo. Por lo anterior, el objetivo de la presente investigación fue estudiar el estado nutrimental de ocho genotipos de jamaica en etapa de floración, mediante el análisis químico en ECP, lecturas SPAD y análisis químico de tejido vegetal con métodos de laboratorio.

\section{MATERIALES Y MÉTODOS}

Mediante recorridos de campo de diciembre de 2016 a febrero de 2017 en los municipios de Apaxtla de Castrejón ( $18^{\circ} 08^{\prime}$ 00" LN y $99^{\circ} 56^{\prime} 05^{\prime \prime}$ LO), Ayutla de los Libres ( $16^{\circ} 57^{\prime} 57^{\prime \prime}$ LN y $99^{\circ} 05^{\prime} 38^{\prime \prime}$ LO) y Tecoanapa ( $18^{\circ} 14^{\prime} 16.44^{\prime \prime}$ LN y $95^{\circ} 59^{\prime} 41.17^{\prime \prime}$ LO), en Guerrero, México, respectivamente; donde se seleccionaron plantas y se recolectaron semillas de genotipos de jamaica (Hisbiscus sabdariffa L.) cultivadas en campo. Las plantas se clasificaron de acuerdo con sus características físicas del tallo y cáliz, asignando claves de identificación según el genotipo (Tabla 1). Para posteriormente almacenar las semillas en frascos de vidrio a temperatura ambiente de marzo a junio del mismo año. La siembra se realizó en forma directa el 6 de julio de 2017, en bolsas de polietileno negro de $12 \mathrm{~L}$, llenadas con una mezcla de tezontle fino (tamaño de partícula menor a $2 \mathrm{~mm}$ ) y tierra de monte (suelo luvisol) (relación $1: 1 \mathrm{v} / \mathrm{v})$. Las semillas se colocaron a una profundidad aproximada de $5 \mathrm{~cm}$ y se cubrieron con el mismo sustrato.

Las macetas fueron distribuidas aleatoriamente dentro de un invernadero tipo cenital cubierto con plástico de color blanco lechoso $(800 \mu \mathrm{m})$ con $70 \%$ de transmitancia de luz y temperatura media durante el ciclo del cultivo de $31^{\circ} \mathrm{C}$, ubicado en la Facultad de Ciencias Agropecuarias y Ambientales, unidad Tuxpan, de la Universidad Autónoma de Guerrero, en las coordenadas: $18^{\circ} 20^{\prime} 51^{\prime \prime} \mathrm{LN}$ y $99^{\circ} 30^{\prime} 32^{\prime \prime} \mathrm{LO}$; a $758 \mathrm{~m}$ de altitud. Posteriormente, las macetas se regaron con agua corriente para favorecer la germinación, la cual se presentó entre los tres y cinco días después de la siembra (dds). A los 15 dds se realizó un raleo, dejando una planta por maceta.

El riego del cultivo se realizó con un sistema de goteo, que consistió en emisores de $8 \mathrm{~L} \mathrm{~h}^{-1}$ con distribuidores localizados en la base de las plantas. Se realizaron dos riegos de 40 minutos cada uno, por la mañana (10:00 h) y tarde (18:00 h). La fertilización se realizó de acuerdo a lo sugerido por Alejo (2016), con 44-30-20 $\mathrm{kg} \mathrm{ha}^{-1}$ de NPK, en dos etapas; la primera fertilización (50\%) se realizó a los 33 dds y la segunda $(50 \%)$ a los 76 dds. El diseño experimental consistió en un diseño completamente al azar, con ocho tratamientos y cinco repeticiones, considerando como tratamiento a cada genotipo, la unidad experimental estuvo constituida por una maceta con una planta.

A los 135 dds (etapa de floración) se registraron las lecturas SPAD en hojas recientemente maduras seleccionadas aleatoriamente en cada uno de los genotipos, con un SPAD (Soil Plant Analysis Development) Minolta 502, con base en la metodología propuesta por González et al. (2013) y Cruz et al. (2017), que consiste en obtener el promedio de tres lecturas en lugares distintos de la hoja (proximal, media y distal). Así mismo, se determinaron los iones $\mathrm{NO}_{3^{-}}, \mathrm{K}^{+}, \mathrm{Ca}^{2+}$ y $\mathrm{Na}^{+}$en el extracto celular de peciolos (ECP) (Damián-Nava et al. 2017). Los pecíolos fueron obtenidos de hojas recientemente maduras muestreadas entre las 10:00 y 13:00 h (Pino et al. 2012). La determinación se realizó con medidores portátiles (ionómetros) tipo Cardy (Horiba ${ }^{\circledR}$ ) LAQUAtwin, previamente calibrados con solución estándar, se agregaron de dos a tres gotas de ECP en el equipo respectivo para la determinación del ion correspondiente.

La lámina foliar y el sobrante de los pecíolos fueron recolectados y depositados en bolsas de papel previamente perforadas y posteriormente colocadas en una estufa de secado con aire circulante durante $72 \mathrm{~h}$ a $75^{\circ} \mathrm{C}$, hasta que las muestras se mantuvieron a peso constante. Las muestras fueron molidas con un molino de acero inoxidable y de acuerdo con la metodología descrita por Alcántar y Sandoval (1999), se sometieron a una digestión húmeda a través de una mezcla de ácido sulfúrico y perclórico. La determinación de nitrógeno $(\mathrm{N})$ se cuantificó por el método 
Tabla 1. Características generales de los genotipos de jamaica recolectados en campo.

\begin{tabular}{lll}
\hline Genotipos & Procedencia & Características generales de la planta \\
\hline G8: Sudán & Apaxtla & Tipo Sudán negra: tallos color rojo intenso, cálices grandes de color rojo oscuro. \\
G9: Tecoanapa & Ayutla de los libres & Tipo Tecoanapa: tallos color rojo, cálices pequeños de color rojo. \\
G12: Tecoanapa & Ayutla de los libres & Tipo Tecoanapa: tallos color rojo, cálices pequeños de color rosa/rojizo. \\
G17: Sudán & Tecoanapa & Tipo Sudán roja: tallos color rojo, cálices medianos color rojo oscuro. \\
G20: Tecoanapa & Tecoanapa & Tipo Tecoanapa: tallos color rojo/café, cálices pequeños de color rojo \\
G28: Sudán & Tecoanapa & Tipo Sudán negra: tallos de color rojo oscuro, cálices grandes de color rojo oscuro. \\
G40: Sudán & Tecoanapa & Tipo Sudán roja: tallos de color rojo oscuro, cálices grandes de color rojo. \\
Var Tecoanapa & Tecoanapa & Tipo Tecoanapa: tallos de color rojos, cálices pequeños de color rojo. \\
\hline ॠVariedad Tecoanapa (número de registro: JAM-004-260210).
\end{tabular}

Kjeldahl (Bremner 1965). Los macronutrimentos (fósforo $(\mathrm{P})$, potasio $(\mathrm{K})$, calcio $(\mathrm{Ca})$ y magnesio $(\mathrm{Mg})$ ) y micronutrimentos (hierro $(\mathrm{Fe})$, cobre $(\mathrm{Cu})$, zinc $(\mathrm{Zn})$, manganeso (Mn), y boro (B)) se cuantificaron con espectroscopia de emisión atómica de inducción por plasma (ICP-AES) (Agilent ICP-AES, Modelo 725-ES, Victoria, Australia).

Se realizaron los análisis de varianza de los datos mediante el programa estadístico SAS (Statistical Analysis System) versión 9.3, y los resultados que presentaron diferencias estadísticas se les aplicó una prueba de comparación de medias de Tukey $(\alpha=$ 0.05). Además, se realizó un análisis de correlación de Pearson $(\alpha=0.05)$ entre variables significativas.

\section{RESULTADOS}

\section{lones en ECP y lecturas SPAD}

Los valores de los iones detectados en el análisis de ECP mostraron diferencias significativas para las concentraciones de $\mathrm{NO}_{3^{-}}, \mathrm{Ca}^{2+}$ y Na${ }^{+}$(Tabla 2). Las mayores concentraciones de $\mathrm{NO}_{3}$ - se encontraron en los genotipos G20 y las menores en G8, G12, G40 y Var. Tecoanapa. La concentración de $\mathrm{Ca}^{2+}$ fue superior en G40, seguida por G28 y después el resto de los genotipos. Respecto al $\mathrm{Na}^{+}$, la mayor concentración se tuvo en el G40 y el más bajo en el G17. Con respecto a las lecturas SPAD detectadas en los genotipos de jamaica estudiados también presentaron diferencias significativas. El G40 registró el menor valor mientras que G28 fue el que presentó el mayor. El resto de los genotipos tuvieron valores similares.

\section{Concentración de macronutrimentos}

La concentración de macronutrimentos en las hojas fue diferente en los genotipos de jamaica estudiados (Tabla 3). La mayor concentración de N, P y Mg se presentó en G28 y de $\mathrm{N}$ en Var Tecoanapa. Sin embargo, el Ca fue mayor en G28, G40 y en Var Tecoanapa, esta última también registró la mayor concentración de K. Por el contrario, la menor concentración de N la tuvieron G8, G9, G17 y G20, respectivamente. Mientras que los genotipos G9 y G8 mostraron menor acumulación de P. Cabe agregar que G40 tuvo menor concentración de K y G9 registró menor acumulación de $\mathrm{Ca}$ y $\mathrm{Mg}$.

\section{Concentración de micronutrimentos}

Se tuvieron diferencias en las concentraciones de micronutrimentos en las hojas de los genotipos de jamaica estudiados (Tabla 4). Se encontró que las mayores concentraciones de $\mathrm{Fe}, \mathrm{Mn}$ y $\mathrm{B}$ las registró el G12 con 190.50, 163.57, 388.11 y $50.48 \mathrm{mg} \mathrm{kg}^{-1}$, respectivamente. Mientras que la acumulación de $\mathrm{Cu}$, los valores mayores fueron para la Var Tecoanapa y los menores fueron para G9. Con respecto, a la concentración de Zn, G40 presentó los mayores valores, mientras que G8 y G12 presentaron menor acumulación de este elemento.

\section{Correlación entre lecturas SPAD, concentración de nutrimentos en ECP y en tejido vegetal}

En la mayoría de los genotipos estudiados se encontraron valores bajos de $r^{2}$ entre las lecturas SPAD en las hojas y los iones específicos en el ECP (Tabla 5). Una excepción de las lecturas SPAD y la concentración de $\mathrm{Na}^{+}$se encontró en los genotipos G8 $\left(r^{2}=0.60\right), G 28\left(r^{2}=0.67\right)$ y $G 40\left(r^{2}=0.60\right)$, 
Flores-Bernal et al.

Tabla 2. Concentración de iones específicos en el extracto de savia en pecíolo y lecturas SPAD en hojas de ocho genotipos de jamaica en floración.

\begin{tabular}{|c|c|c|c|c|c|}
\hline \multirow{2}{*}{ Genotipo } & $\mathrm{NO}_{3-}^{-}$ & $\mathrm{K}^{+}$ & $\mathrm{Ca}^{2+}$ & $\mathrm{Na}^{+}$ & \multirow[t]{2}{*}{ Lecturas SPAD } \\
\hline & \multicolumn{4}{|c|}{$\mathrm{mg} \mathrm{L}^{-1}$} & \\
\hline G8 & $436.00^{c}$ & $2180.00^{a}$ & $3.80^{b}$ & $70.80^{a b}$ & $46.20^{a b}$ \\
\hline G9 & $456.00^{b c}$ & $2100.00^{a}$ & $4.40^{b}$ & $61.00^{a b c}$ & $44.99^{b}$ \\
\hline G12 & $332.00^{c}$ & $1722.00^{a}$ & $4.40^{b}$ & $53.00^{a b c}$ & $47.19^{a b}$ \\
\hline G17 & $522.00^{b c}$ & $2380.00^{a}$ & $3.60^{b}$ & $42.40^{c}$ & $45.14^{b}$ \\
\hline G20 & $680.00^{a b}$ & $2680.00^{a}$ & $3.80^{b}$ & $45.40^{b c}$ & $49.48^{a b}$ \\
\hline G28 & $872.00^{a}$ & $2620.00^{a}$ & $6.20^{a b}$ & $50.80^{a b c}$ & $51.71^{a}$ \\
\hline G40 & $392.00^{c}$ & $2120.00^{a}$ & $8.00^{a}$ & $76.80^{a}$ & $38.58^{c}$ \\
\hline Var Tecoanapa & $334.00^{c}$ & $2740.00^{a}$ & $4.40^{b}$ & $62.20^{a b c}$ & $50.17^{a b}$ \\
\hline DMS & 234.17 & 1049.00 & 2.97 & 27.99 & 6.12 \\
\hline
\end{tabular}

Valores con la misma letra en la misma columna son estadísticamente iguales, de acuerdo con la prueba de Tukey $(\alpha=0.05)$. DMS: diferencia mínima significativa, G: genotipo, SPAD: Soil Plant Analysis Development. *Variedad Tecoanapa (número de registro: JAM-004-260210).

Tabla 3. Concentración de macronutrimentos en hojas de ocho genotipos de jamaica en etapa de floración.

\begin{tabular}{|c|c|c|c|c|c|}
\hline \multirow{2}{*}{ Genotipo } & $\mathrm{N}$ & $\mathrm{P}$ & $\mathrm{K}$ & $\mathrm{Ca}$ & $\mathrm{Mg}$ \\
\hline & $\%$ & \multicolumn{4}{|c|}{$\mathrm{mg} \mathrm{kg}^{-1}$} \\
\hline G8 & $1.41 \pm 0.05^{d e}$ & $954.02 \pm 26.46^{d e}$ & $9631.30 \pm 47.11^{c d}$ & $12806.80 \pm 48.01^{c}$ & $2768.10 \pm 10.40^{d}$ \\
\hline G12 & $1.77 \pm 0.02^{b c}$ & $1149.15 \pm 42.52^{c}$ & $9485.50 \pm 32.24^{c d}$ & $14385.20 \pm 6.22^{a b}$ & $2414.15 \pm 6.96^{e}$ \\
\hline G17 & $1.33 \pm 0.04^{e}$ & $1050.15 \pm 26.21^{c d}$ & $10238.20 \pm 433.49^{c d}$ & $13435.60 \pm 423.06^{b c}$ & $3443.09 \pm 65.97^{b}$ \\
\hline G20 & $1.65 \pm 0.05^{d e}$ & $1424.58 \pm 15.14^{b}$ & $10473.60 \pm 81.39^{c}$ & $12503.00 \pm 135.06^{c}$ & $2444.15 \pm 25.45^{e}$ \\
\hline G40 & $1.70 \pm 0.07^{b c}$ & $1120.92 \pm 25.52^{c}$ & $8649.10 \pm 711.68^{d}$ & $14884.70 \pm 142.27^{a b}$ & $2984.60 \pm 3.01^{c}$ \\
\hline Var Tecoanapa & $1.93 \pm 0.15^{a b}$ & $1526.12 \pm 29.30^{b}$ & $19051.40 \pm 784.47^{a}$ & $15132.00 \pm 885.51^{a}$ & $2259.48 \pm 4.57^{f}$ \\
\hline DMS & 0.28 & 110.18 & 1710.80 & 1498.30 & 105.26 \\
\hline
\end{tabular}

Valores con la misma letra en la misma columna son estadísticamente iguales, de acuerdo con la prueba de Tukey ( $\alpha=0.05)$. DMS: diferencia mínima significativa; G: genotipo. * Variedad Tecoanapa (número de registro: JAM-004-260210).

Tabla 4. Concentración de micronutrimentos en las hojas de ocho genotipos de jamaica en floración.

\begin{tabular}{|c|c|c|c|c|c|}
\hline \multirow{2}{*}{ Genotipo } & $\mathrm{Fe}$ & $\mathrm{Cu}$ & $\mathrm{Zn}$ & $\mathrm{Mn}$ & B \\
\hline & \multicolumn{5}{|c|}{$\mathrm{mg} \mathrm{kg}^{-1}$} \\
\hline G8 & $115.30 \pm 0.92^{e}$ & $2.56 \pm 0.26^{b c}$ & $5.92 \pm 1.83^{c}$ & $360.12 \pm 2.34^{b}$ & $35.70 \pm 0.06^{d}$ \\
\hline G9 & $163.57 \pm 6.56^{b c}$ & $2.32 \pm 0.24^{c}$ & $10.45 \pm 1.99^{a b c}$ & $265.69 \pm 0.17^{c d}$ & $36.62 \pm 0.52^{d}$ \\
\hline G12 & $190.50 \pm 1.14^{a}$ & $3.04 \pm 0.17^{a b c}$ & $8.33^{b} \pm 0.39^{c}$ & $388.11 \pm 3.76^{a}$ & $50.48 \pm 0.18^{a}$ \\
\hline G17 & $125.36 \pm 1.57^{e}$ & $3.69 \pm 0.79^{a b}$ & $14.47 \pm 0.26^{a b}$ & $252.73 \pm 1.06^{d}$ & $42.35 \pm 0.83^{c}$ \\
\hline G20 & $148.92 \pm 5.54^{c d}$ & $3.07 \pm 0.18^{a b c}$ & $15.00 \pm 0.18^{a b}$ & $216.31 \pm 15.08^{e}$ & $41.42 \pm 0.55^{c}$ \\
\hline G28 & $169.97 \pm 2.58^{b}$ & $3.76 \pm 0.14^{a b}$ & $8.64 \pm 0.91^{a b c}$ & $259.36 \pm 0.77^{c d}$ & $31.60 \pm 0.01^{e}$ \\
\hline G40 & $128.12 \pm 8.69^{e}$ & $2.95 \pm 0.09^{b c}$ & $15.94 \pm 1.14^{a}$ & $277.98 \pm 0.96^{c}$ & $46.03 \pm 1.11^{b}$ \\
\hline Var Tecoanapa & $129.70 \pm 5.73^{d e}$ & $4.17 \pm 0.23^{a}$ & $10.05 \pm 1.33^{a b c}$ & $179.38 \pm 0.60^{f}$ & $46.54 \pm 0.81^{b}$ \\
\hline DMS & 19.45 & 1.31 & 7.30 & 22.13 & 2.50 \\
\hline
\end{tabular}

Valores con la misma letra en la misma columna son estadísticamente iguales, de acuerdo con la prueba de Tukey $(\alpha=0.05)$. DMS: diferencia mínima significativa, G: genotipo. * Variedad Tecoanapa (número de registro: JAM-004260210).

cuyos coeficientes de correlación fueron moderados. En cambio, las lecturas SPAD y $\mathrm{K}^{+}$en los genotipos G9 y G12, presentaron una correlación negativa de moderada a alta, con valores de $r^{2}=-0.70$ y -0.82 , respectivamente. El mismo comportamiento se presentó al relacionar las lecturas SPAD con $\mathrm{Ca}^{2+}$, los cuales mostraron una alta correlación negativa en los genotipos $\mathrm{G} 12\left(\mathrm{r}^{2}=-0.71\right)$ y $\mathrm{G} 17\left(\mathrm{r}^{2}=-0.87\right)$.

Por otro lado, al correlacionar los valores de las concentraciones de iones en el ECP y las lecturas SPAD, con las concentraciones de $\mathrm{N}$ total, $\mathrm{K}^{+}$ y $\mathrm{Ca}^{2+}$ determinadas en laboratorio en las hojas de jamaica (Tabla 6), se tuvieron bajos coeficientes de correlación, sobre todo entre el $\mathrm{N}$ total con la concen- 
Tabla 5. Correlaciones cruzadas entre lecturas SPAD y iones específicos en ECP en genotipos de jamaica en etapa de floración.

\begin{tabular}{crrrr}
\hline Genotipo & SPAD-NO $_{3-}$ & SPAD-K $^{+}$ & SPAD-Ca $^{2+}$ & SPAD-Na $^{+}$ \\
\hline G8 & 0.00 & 0.10 & 0.00 & 0.60 \\
G9 & -0.10 & -0.70 & -0.41 & 0.30 \\
G12 & -0.56 & -0.82 & -0.71 & -0.40 \\
G17 & -0.05 & 0.56 & -0.87 & 0.20 \\
G20 & -0.05 & -0.10 & -0.35 & -0.40 \\
G28 & -0.21 & -0.46 & 0.05 & 0.67 \\
G40 & 0.20 & 0.10 & -0.45 & 0.60 \\
Var Tecoanapa & -0.30 & -0.10 & -0.36 & 0.40 \\
\hline G: genotipo. * Variedad Tecoanapa (número de registro: JAM-004-260210).
\end{tabular}

Tabla 6. Coeficientes de correlación entre iones en ECP, SPAD y concentración de macronutrimentos en hojas de ocho genotipos de jamaica en etapa de floración.

\begin{tabular}{|c|c|c|c|c|c|}
\hline \multirow{2}{*}{$\begin{array}{l}\text { Concentración de } \\
\text { nutrimentos en hoja }\end{array}$} & \multicolumn{4}{|c|}{ lones en el extracto celular de pecíolos } & \multirow{2}{*}{$\begin{array}{l}\text { Lecturas } \\
\text { SPAD }\end{array}$} \\
\hline & $\mathrm{NO}_{3-}$ & $\mathrm{K}^{+}$ & $\mathrm{Ca}^{2+}$ & $\mathrm{Na}^{+}$ & \\
\hline $\mathrm{N}$ total & 0.16 & 0.50 & 0.64 & 0.05 & 0.42 \\
\hline $\mathrm{K}$ & 0.37 & 0.61 & -0.11 & -0.37 & 0.35 \\
\hline $\mathrm{Ca}$ & 0.07 & 0.49 & 0.72 & 0.14 & -0.06 \\
\hline
\end{tabular}

tración de $\mathrm{NO}_{3-}$ y $\mathrm{Na}^{+}$en ECP. El mismo comportamiento se tuvo al correlacionar las lecturas SPAD con la concentración de $\mathrm{N}$ en hoja, la cual también fue baja con $r^{2}=0.42$. Sin embargo, se observó que el $\mathrm{N}$ total presentó coeficientes de correlación moderados con el $\mathrm{K}^{+}$y el $\mathrm{Ca}^{2+}$ detectados en el ECP. El mismo comportamiento tuvo el $\mathrm{K}$ en el tejido seco de hojas, con el $\mathrm{K}^{+}$en el ECP, al presentar una correlación moderada $\left(r^{2}=0.61\right)$. Por otro lado, la concentración de Ca en las hojas tuvo mayor correlación (0.72) con el $\mathrm{Ca}^{2+}$ en el ECP en las hojas de los genotipos de jamaica estudiados en el presente trabajo.

\section{DISCUSIÓN}

\section{lones en ECP y lecturas SPAD}

No se encontraron valores de referencia de iones específicos de ECP en hojas de jamaica. Pero los resultados encontrados en el presente trabajo se aproximan a los valores de iones en ECP reportados en otras especies como jitomate (Solanum licopersicum L.), cuyas concentraciones oscilan de 218.20 a $618.20 \mathrm{mg} \mathrm{L}^{-1}$ de $\mathrm{NO}_{3^{-}}$(Preciado et al. 2011). Aunque los resultados de esta investigación son bajos comparados con lo reportado por Romero et al. (2013), quienes encontraron concentraciones superiores de $\mathrm{NO}_{3^{-}}\left(12750 \mathrm{mg} \mathrm{L}^{-1}\right)$ y K $\mathrm{K}^{+}$(10 100 $\mathrm{mg} \mathrm{L}^{-1}$ ) en el ECP de hojas de menta (Menta piperita L.). Mientras que Damián et al. (2017) reportaron valores de $\mathrm{NO}_{3^{-}}$de 2144 a $3778 \mathrm{mg} \mathrm{L}^{-1}, \mathrm{~K}^{+}$de 2642 a $2648 \mathrm{mg} \mathrm{L}^{-1}, \mathrm{Ca}^{2+}$ de 200 a $1496 \mathrm{mg}$ $\mathrm{L}^{-1}$ y Na${ }^{+}$de 1321.7 a $1783 \mathrm{mg} \mathrm{L}^{-1}$, en ECP de hojas de aguacate (Persea americana Mill) con fertilización orgánica. Mientras que Piedragil-Ocampo et al. (2019) mencionaron valores que oscilaron de 86.00 a $490 \mathrm{mg} \mathrm{L}^{-1} \mathrm{de} \mathrm{NO}_{3^{-}}$, de 134.11 a $280.50 \mathrm{mg}$ $\mathrm{L}^{-1} \mathrm{de} \mathrm{K}^{+}$, de 5.75 a $25.71 \mathrm{mg} \mathrm{L}^{-1}$ de $^{2+} \mathrm{Ca}^{2+}$ y de 8.83 a $28.79 \mathrm{mg} \mathrm{L}^{-1}$ de $\mathrm{Na}^{+}$en ECP en variedades de mango amarillo (Manguifera indica L.). Las diferencias en la concentración de los iones encontrados se atribuyen principalmente a la variabilidad genotípica, cuyas características fenotípicas provocan grandes variaciones en el contenido de nutrimentos en el ECP y en tejido vegetal por la diferente capacidad de absorción de nutrientes que tiene cada genotipo (Pino et al. 2012). También se ha observado mayor acumulación de $\mathrm{NO}_{3-}$ y $\mathrm{Ca}^{2+}$ y disminución en la concentración de $\mathrm{K}^{+}$en ECP con el aumento de la edad de la planta (Noh-Medina et al. 2010). Así mismo, la variación entre la concentración de nutrientes el ECP puede atribuirse a las condiciones climáticas y a la actividad química que persiste en la solución en el medio de crecimiento radical (Llanderal et al. 2020). 
Por otro lado, las lecturas SPAD detectadas coinciden con los valores reportados en el cultivo de jamaica por Fahmy y Hassan (2019), con lecturas SPAD que oscilan de 35.33 a 46.00 , dichos valores varían de acuerdo con la época de producción, las dosis y fuentes de fertilización, lo que modifica el contenido de clorofila y la concentración de los nutrimentos en las hojas (Sánchez-García et al. 2016, Torres et al. 2017, Al-Sayed et al. 2020). Sin embargo, diversos estudios han demostrado que las lecturas SPAD pueden ser afectadas por el genotipo o cultivar dentro de la misma especie (Piedragil et al. 2019, Santillano et al. 2019).

\section{Concentración de macronutrimentos}

La concentración de macronutrimentos encontrados en las hojas de jamaica, variaron de acuerdo con el genotipo. Al respecto, Eman et al. (2007) reportaron valores de 2.09 a $2.86 \%$ para $\mathrm{N}$, de 0.39 a $0.64 \%$ para $\mathrm{P}$, y de 1.78 a $2.04 \%$ de $\mathrm{K}$, en hojas de jamaica, dichos valores superan a los encontrados en la presente investigación. Por su parte, Musa y Ogbadoyi (2012) detectaron valores bajos de $\mathrm{Ca}(22.52$ a $\left.24.81 \mathrm{mg} \mathrm{kg}^{-1}\right), \mathrm{Mg}\left(17.19\right.$ a $\left.20.79 \mathrm{mg} \mathrm{kg}^{-1}\right)$ y $\mathrm{K}$ (36.01 a $43.33 \mathrm{mg} \mathrm{kg}^{-1}$ ) en hojas de jamaica en estado vegetativo y reproductivo. Sin embrago, a excepción del $\mathrm{P}$, los promedios de las concentraciones de N, K, Ca y Mg determinadas en este estudio se aproximan a lo afirmado por Salisbury y Ross (1994), quienes indican concentraciones en el tejido seco de plantas superiores de $1.5 \%$ para N, 1.0, 0.5, 0.2 y $0.2 \%$ para $\mathrm{K}, \mathrm{Ca}, \mathrm{P}$ y $\mathrm{Mg}$, respectivamente. Por otro lado, Sánchez-Prado et al. (2019) reportaron valores de $2.20,0.16,1.55,2.82$, Ca y $0.29 \mathrm{~g}$ planta $^{-1}$ de N, P, K, Ca y Mg, respectivamente, acumulados en hojas de jamaica variedad UAN-6, dichas variaciones se atribuyen al manejo de la fertilización y variedades; los mismos autores indican que en las etapas productivas se registra mayor concentración de $\mathrm{N}$ y $\mathrm{K}$, mientras que el $\mathrm{P}$ y $\mathrm{Mg}$ son nutrimentos que se requieren en menor cantidad.

\section{Concentración de micronutrimentos}

Con respecto a la concentración de micronutrimentos, Eman et al. (2007) reportaron en hojas de jamaica concentraciones superiores de $\mathrm{Cu}(42.2$ a $56.4 \mathrm{mg} \mathrm{kg}^{-1}$ ) y $\mathrm{Zn}\left(51.6\right.$ a $\left.72.0 \mathrm{mg} \mathrm{kg}^{-1}\right)$, e inferiores de $\mathrm{Mn}$ (57.2 a $88.2 \mathrm{mg} \mathrm{kg}^{-1}$ ), en comparación con los valores detectados en el presente estudio, aunque el contenido de Fe (132 a $162 \mathrm{mg} \mathrm{kg}^{-1}$ ) indicado por estos autores, fue similar. Por el contrario, Musa y Ogbadoyi (2012) determinaron valores inferiores de $\mathrm{Fe}$ (33.17 a $35.20 \mathrm{mg} \mathrm{kg}^{-1}$ ), Zn (0.03 a $0.04 \mathrm{mg} \mathrm{kg}^{-1}$ ) y $\mathrm{Cu}\left(1.88\right.$ a $2.89 \mathrm{mg} \mathrm{kg}^{-1}$ ) en hojas de jamaica, al respecto se sabe que dichos valores varían de acuerdo con el estado fenológico de la planta (vegetativo y reproductivo). Con excepción del $\mathrm{Cu}$ y $\mathrm{Zn}$, la concentración de $\mathrm{Fe}, \mathrm{Mn}$ y $\mathrm{B}$ superan los valores reportados por Salisbury y Ross (1994), quienes señalan valores de 100.0, 6.0, 20.0, 50.0 y $20.0 \mathrm{mg} \mathrm{kg}^{-1}$ para $\mathrm{Fe}, \mathrm{Cu}, \mathrm{Zn}, \mathrm{Mn}$ y B, respectivamente. Estos valores pueden variar de acuerdo con la edad de la planta, la concentración de otros elementos minerales, las condiciones ambientales en las que se desarrolla el cultivo, la variedad o genotipo, y con el manejo agronómico del cultivo (Marschner 2012, Pino et al. 2012).

\section{Correlación entre lecturas SPAD, concentración de nutrimentos en ECP y en tejido vegetal}

Los valores de $\mathrm{r}^{2}$ encontrados entre $\mathrm{NO}_{3}$, lecturas SPAD y los valores $\mathrm{N}$ en tejido; indican que la concentración de $\mathrm{N}$ en las hojas no se asoció con los valores detectados con los equipos portátiles. Lo anterior, se atribuye a que elementos como el $\mathrm{N}$ están condicionados al contenido en tejido seco, lo que influye en las bajas concentraciones en el extracto celular (Noh-Medina et al. 2010). Lo que pudo afectar la concentración de $\mathrm{NO}_{3^{-}}$en los peciolos de las hojas. Posiblemente, en la etapa en la que se realizó el muestreo, el $\mathrm{N}$ suministrado por el sustrato y la fertilización ya había sido incorporado a estructuras orgánicas como lo señala Marschner (2012), como componente de la clorofila (Núñez et al. 2017). También puede deberse a un desequilibrio por mal funcionamiento de la enzima nitrato reductasa (Cadahía 2008, Sánchez-García et al. 2016), esto a pesar que existen reportes que el ECP representa bien el estado nutricional del $\mathrm{N}$, sobre todo cuando se tienen niveles de abastecimiento de $\mathrm{N}$ elevados, como lo observó 
Pino et al. (2012) en el cultivo de la vid, reportando valores de $r^{2}$ de 0.74 . Además, estos autores indican que valores bajos de $r^{2}$ se deben a un alto coeficiente de variación del $\mathrm{NO}_{3^{-}}$que afectan su relación con el $\mathrm{N}$ foliar.

A pesar que en otros cultivos se ha observado que a medida que se incrementa el $\mathrm{N}$, se incrementan las lecturas SPAD (Ribeiro et al. 2015, Mendoza-Tafolla et al. 2019), en la presente investigación se documentó lo contrario. Adicionalmente, los coeficientes de correlación moderados entre el $\mathrm{N}$ con el $\mathrm{K}^{+}$y $\mathrm{Ca}^{2+}$ detectados en el ECP, se debe a que el $\mathrm{Ca}$ y el $\mathrm{K}$ se acumulan en mayores cantidades dentro de la planta en las etapas productivas. En el caso del Ca la acumulación es con grupos oxalatos o carboxílicos de pectinas de las paredes celulares, mientras que el $\mathrm{K}$ forma estructuras y es transportador de fotosintatos (Marschner 2012). Además, es común encontrar relaciones antagónicas entre algunos elementos que provocan desequilibrios dentro de la planta (Sánchez-García et al. 2016). Mientras que la correlación moderada entre el $\mathrm{K}$ en tejido de hojas con el $\mathrm{K}^{+}$en el ECP, se debe a que en el cultivo de jamaica el $\mathrm{K}$ es el elemento que más se acumula en el tejido en la etapa reproductiva, como lo mencionaron Sánchez-Prado et al. (2019). Pero hay reportes que el $\mathrm{K}$ en el ECP puede no presentar correlación significativa con el $\mathrm{K}^{+}$de la hoja (Pino et al. 2012), debido a que en la etapa reproductiva de la jamaica este elemento se transloca a la formación de flores y frutos (cápsulas), disminuyendo su concentración en el resto de la planta (Sánchez-Prado et al. 2019). En contraste, la alta correlación detectada entre la concentración de $\mathrm{Ca}$ en tejido vegetal y el $\mathrm{Ca}^{2+}$ en el ECP de los genotipos de jamaica estudiados, se atribuye a que este elemento aumenta conforme se incrementa la edad de la planta, acumulando altos contenidos de $\mathrm{Ca}$ en la biomasa, lo que provoca bajos niveles de otros nutrimentos como N, P, K, Mg, $\mathrm{Cu}, \mathrm{Fe}$ y Mn (Noh-Medina et al. 2010). Además, la concentración de este elemento en los pecíolos varía de acuerdo a la estación en la que se establece el cultivo (Lara et al. 2019). Lo anterior, reafirma que cada variedad puede responder de manera diferente durante la absorción de un nutriente, mientras que, a la inversa, los factores ambientales pueden influir en dicha absorción (Ávila-Juárez y Rodríguez-Ruíz 2020). Sin embargo, las relaciones encontradas entre la concentración de nutrimentos en tejido seco y el extracto celular de peciolo pueden ser consideradas como un principio en la búsqueda de los niveles óptimos de referencia (Noh-Medina et al. 2010), para los genotipos de jamaica utilizados en la presente investigación.

\section{CONCLUSIONES}

El genotipo influyó en la concentración de iones en el ECP, en las lecturas SPAD y en la concentración de nutrimentos en hojas de jamaica en etapa de floración. A pesar que los valores de los iones del ECP y lecturas SPAD tuvieron baja correlación con la concentración de nutrimentos en tejido seco, estos son un indicador de la actividad química del pecíolo y la hoja de este cultivo durante la floración en los genotipos estudiados, sobre todo al relacionar lecturas SPAD con $\mathrm{K}^{+}$y $\mathrm{Ca}^{2+}$ en $\mathrm{G} 9, \mathrm{G} 12$ y G17, así como el $\mathrm{Ca}^{+2}$ en el ECP y Ca en las hojas. Los valores detectados de $\mathrm{NO}_{3^{-}}, \mathrm{K}^{+}, \mathrm{Ca}^{2+}$ y $\mathrm{Na}^{+}$en el ECP, lecturas SPAD en hojas y nutrimentos en tejido vegetal, pueden ser usados como valores de referencia para estudios posteriores y aplicados en la determinación de dosis de fertilización en cada uno de los genotipos de jamaica estudiados.

\section{AGRADECIMIENTOS}

Al Programa para el Desarrollo Profesional Docente (PRODEP-SEP) por el financiamiento de la presente investigación a través del proyecto PRODEPSEP 511-6/17-7665.

\section{LITERATURA CITADA}

Alcántar GG, Sandoval VM (1999) Manual de análisis químico de tejido vegetal. Publicación especial 10. So- 
ciedad Mexicana de la Ciencia del Suelo. Chapingo, Estado de México. 156p.

Alejo JA (2016) Cultivo de jamaica en dos sistemas de producción en Guerrero. Folleto para productores número 16. Instituto Nacional de Investigaciones Forestales, Agrícolas y Pecuarias. Iguala de la independencia Guerrero. 21p.

Al-Sayed HM, Hegab SA, Youssef MA, Khalafalla MY, Almaroai YA, Ding Z, Eissa MA (2020) Evaluation of quality and growth of roselle (Hibiscus sabdariffa L.) as affected by bio-fertilizers. Journal of Plant Nutrition 43: 1025-1035.

Ávila-Juárez L, Rodríguez-Ruiz MA (2020) Rapid NPK diagnosis in tomato using petiole sap analysis with the DRIS method. Horticultura Brasileira 38: 306-311.

Bremner JM (1965) Total nitrogen. In: Black CA (ed). Methods of soil analysis. Part 2. Agronomy 9. American Society of Agronomy. Madison, WI, USA. pp: 1149-1178.

Cadahía LC (2008) La savia como índice de fertilización. Cultivos agroenergéticos, hortícolas, frutales y ornamentales. Ediciones Mundi Prensa. Madrid. 255p.

Castro-Brindis R, Sánchez GP, Peña LA, Alcántar GG, Baca CGA, López RRM (2000) Nitratos en el extracto celular de pecíolos y tallo de tomate de cáscara (Physalis ixocarpa Brot.) y su relación con el rendimiento. Revista Chapingo Serie Horticultura 61: 33-38.

Cruz CE, Can CA, Loera RLJ, Aguilar BG, Pineda PJ, Bulgarín MR (2017) Extracción de N-P-K en Coriandrum sativum "Pakistan" en hidroponia. Revista Mexicana de Ciencias Agrícolas 8: 355-367.

Damián NA, Arellano RL, Hernández CE, Palemón AF, Cruz LB, Vargas AD, Díaz VG, Leiva REI, Ramírez PR (2017) Effect of organic nutrtition in the nursery growth and nutrimental content of native avocados of Ometepec, Guerrero, México. International Journal of Environment, Agriculture and Biotechnology 2: 2732-2739.

Eman EA, Gad A, Badran NM (2007) Effect of cobalt and nickel on plant growth, yield and flavonoids content of Hibiscus sabdariffa L. Australian Journal of Basic and Applied Sciences 1: 73-78.

Etchevers BJD (1999) Técnicas de diagnóstico útiles en la medición de la fertilidad del suelo y el estado nutrimental de los cultivos. Terra Latinoamericana 17: 209-219.

Fahmy AA, Hassan HMS (2019) Influence of different NPK fertilizatión levels and humic acid rates on growth, yield and chemical constituents of roselle (Hibiscus sabdariffa L.). Midddle East Journal of Agriculture Research 8: $1182-1189$.

Gómez SMI, Magnitskiy S, Rodríguez LE (2017) Diagnóstico de $\mathrm{K}^{+}$y $\mathrm{NO}_{3^{-}}$en savia para determinar el estado nutricional en papa (Solanum tuberosum L. subsp. andigena). Revista Colombiana de Ciencias Hortícolas 11: 133-142.

González SKD, Rodríguez MMN, Trejo TLI, García CJL, Sánchez EJ (2013) Efluente y té de vermicompost en la producción de hortalizas de hoja en sistema NFT. Interciencia 38: 863-869.

Lara IAY, Rojas VN, Romero MM, Ramírez THM, Cruz CE, Alcalá JJA, Loredo OC (2019) Crecimiento y acumulación de $\mathrm{NO}_{3-}$ en lechuga hidropónica con relaciones nitrato/amonio en dos estaciones de cultivo. Revista Fitotecnia Mexicana 42: 21-29.

Llanderal QA, García CP, José Pérez AJ, Contreras JI, Segura PML, Reca J, Lao MT (2020) Approach to petiole sap nutritional diagnosis method by empirical model base on climatic and growth parameters. Agronomy 10(2). DOI: 10.3390/agronomy10020188.

Marschner P (2012) Mineral nutrition of higher plants. 3th ed. Elsevier. San Diego, California, USA. 651p. 
Mendoza-Tafolla RO, Juárez LP, Ontiveros CRE, Sandoval VM, Alia TI, Alejo SG (2019) Estimating nitrogen and chlorophyll status of romaine lettuce using SPAD and at LEAF readings. Notulae Botanicae Horti Agrobotanici Cluj-Napoca 47: 751-756.

Musa A, Ogbadoyi EO (2012) Effect of nitrogen fertilizer on the levels of some nutrients, anti-nutrients and toxic substances in Hibiscus sabdariffa. Asian Journal of Crop Science 4: 103-112.

Noh-Medina J, Borgues GL, Soria FM (2010) Composición nutrimental de biomasa y tejidos conductores en chile habanero (Capsicum Chinese Jacq.). Tropical and Subtropical Agroecosystems 12: 219-228.

Núñez RF, Grijalva CRL, Roles CF, Macias DR, Escobosa GMI, Santillano CJ (2017) Influencia de la fertirrigación nitrogenada en la concentración de nitratos en el extracto celular de peciolo, el rendimiento y la calidad de tomate en invernadero. Revista de la Facultad de Ciencias Agrarias 49: 93-103.

Piedragil OB, Hernández CE, Mora AJA, Damián NA, Palemón AF, Sarabia RG, Monteón OA (2019) Photosynthetic profiles and nutrimental characterization of yellow mango in México. African Journal of Agricultural Research 14: 467-476.

Pino P, Callejas R, Razeto B, Reginato G (2012) Análisis químico del extracto del extracto peciolar para evaluar el estado nutricional en la vid. Pesquisa Agropecuaria Brasileira 47: 111-117.

Preciado RP, Fortis HM, García HJL, Rueda PE, Esparza RJR, Lara HA, Segura CMA, Orozco VJA (2011) Evaluación de soluciones nutritivas orgánicas en la producción de tomate en invernadero. Interciencia 36: 689-693.

Ribeiro DCA, Katz L, De Pádua AS, Martinez URA (2015) Índice SPAD en el crecimiento y desarrollo de plantas de lisianthus en función de diferentes dosis de nitrógeno en amiente protegido. IDESIA 33: 97-105.

Romero FJC, Rodríguez MMN, Gutierrez CMC, Sánchez EJ (2013) Vermicompost como sustrato en la producción de menta (Mentha piperita L.). Revista Mexicana de Ciencias Agrícolas 4: 889-899.

Salisbury FB, Ross CW (1994) Fisiología vegetal. Grupo Editorial Iberoamericano. México. 687p.

Sánchez-García P, Molinos DSC, Alcántar GG, Sandoval VM (2016) Diagnóstico nutrimental en plantas. En: Alcántar GG, Trejo TLI, GMF (eds). Nutrición de cultivos. Colegio de Postgraduados. Texcoco, Edo. de México, México. pp: 185-238.

Sánchez-Prado JJ, Bulgarín MR, Santiago GA, Juárez RCR, Aburto GCA, Caro VF (2019) Incremento del rendimiento y extracción nutrimental en jamaica mediante soluciones nutritivas. Ecosistemas y Recursos Agropecuarios 6: 1-10.

Santillano CJ, Roque DLG, Núñez RF, Grijalva CRL, Robles CF, Macías DR, Escobosa GI, Cárdenas SV (2019) La fertilidad del suelo afecta el crecimiento, nutrición y rendimiento de algodón cultivado en dos sistemas de riego y diferentes dosis de nitrógeno. Terra Latinoamericana 37: 7-14

Torres BAI, Morales MA, Grijalva CRL, Cervantes DL, Núñez RF (2017) Hierro foliar y acolchado plástico en Capsicum chinense Jacq. Infected wit tospoviruses. Revista Mexicana de Ciencias Agrícolas 8: 369-380. 